\title{
DIMENSION SPECTRUM FOR A NONCONVENTIONAL ERGODIC AVERAGE
}

\author{
YUVAL PERES AND BORIS SOLOMYAK
}

\begin{abstract}
We compute the dimension spectrum of certain nonconventional averages, namely, the Hausdorff dimension of the set of 0,1 sequences, for which the frequency of the pattern 11 in positions $k, 2 k$ equals a given number $\theta \in[0,1]$.
\end{abstract}

\section{INTRODUCTION.}

For a dynamical system $(X, T)$ (say, a continuous self-map of a compact metric space), the dimension spectrum of ordinary Birkhoff averages is defined as the function

$$
\theta \mapsto \operatorname{dim}_{H}\left\{x \in X: \lim _{n \rightarrow \infty} \frac{S_{n} f(x)}{n}=\theta\right\} .
$$

where $S_{n} f(x)=\sum_{k=1}^{n} f\left(T^{k} x\right)$ and $f$ is a function on $X$. It has been widely investigated in Multifractal Analysis, see e.g. [2]. The most basic example of such analysis goes back to Besicovitch [4] and Eggleston [7] who proved that

$$
\operatorname{dim}_{H}\left\{\left(x_{k}\right)_{1}^{\infty} \in\{0,1\}^{\mathbb{N}}: \lim _{n \rightarrow \infty} \frac{1}{n} \sum_{k=1}^{n} x_{k}=\theta\right\}=H(\theta), \quad \theta \in[0,1],
$$

where $H(\theta)=-\theta \log _{2} \theta-(1-\theta) \log _{2}(1-\theta)$ is the entropy function. Throughout the paper, $\{0,1\}^{\mathbb{N}}=\Sigma_{2}$ is the symbolic space, with the usual metric $\varrho\left(\left(x_{k}\right),\left(y_{k}\right)\right)=2^{-\min \left\{n: x_{n} \neq y_{n}\right\}}$. For dimension purposes, this is equivalent to $[0,1]$ with the standard metric, since for any set $A \subset \Sigma_{2}$, its image under the binary representation map has the same dimension as $A$, see [8, Section 2.4].

Furstenberg [10] was the first to consider multiple Birkhoff averages, and their study has become a very active area of research, see e.g. Bourgain [6], Host and Kra [11], and

Date: April 27, 2019.

1991 Mathematics Subject Classification. Primary: 28A80 Secondary: 37C45, 28A78 .

Key words and phrases. multifractal analysis, multiple Birkhoff average, Hausdorff dimension.

The research of B. S. was supported in part by the NSF grant DMS-0968879. 
others. For a system $(X, T)$ one considers

$$
\frac{1}{n} S_{n}\left(f_{1}, \ldots, f_{\ell}\right)(x):=\frac{1}{n} \sum_{k=1}^{n} f_{1}\left(T^{k} x\right) f_{2}\left(T^{2 k} x\right) \cdots f_{\ell}\left(T^{\ell k} x\right)
$$

for some bounded functions $f_{1}, \ldots, f_{\ell}$. Very recently, Yu. Kifer [16] and A.-H. Fan, L. Liao, J. Ma [9] initiated the study of the dimension spectrum for such averages (in [16] more general "nonconventional averages" are considered as well). Multifractal analysis of this kind appears to be very complicated, so it is natural to start with the simplest situation, namely, the shift map $T$ on the symbolic space and the functions $f_{1}, \ldots, f_{\ell}$ depending only on the first digit $x_{1}$, for $\ell \geq 2$. Specializing even further, to $\ell=2$ and $f_{1}(x) \equiv f_{2}(x)=x_{1}$ leads to the sets

$$
A_{\theta}:=\left\{\left(x_{k}\right)_{1}^{\infty} \in \Sigma_{2}: \lim _{n \rightarrow \infty} \frac{1}{n} \sum_{k=1}^{n} x_{k} x_{2 k}=\theta\right\}, \theta \in[0,1] .
$$

The question about the dimension of $A_{\theta}$ was raised in [9]. Note that this directly generalizes the Besicovitch-Eggleston set-up from $\ell=1$ to $\ell=2$.

Motivated by this problem, A.-H. Fan, L. Liao, J. Ma, and J. Schmeling [private communication in August 2010] computed the Minkowski (box-counting) dimension of another set

$$
X_{G}:=\left\{\left(x_{k}\right)_{1}^{\infty} \in \Sigma_{2}: x_{k} x_{2 k}=0 \text { for all } k\right\}
$$

and asked what is its Hausdorff dimension. It is obvious that $X_{G} \subset A_{0}$, and in fact, it is easy to see that $\operatorname{dim}_{H}\left(X_{G}\right)=\operatorname{dim}_{H}\left(A_{0}\right)$.

In joint work with R. Kenyon, we computed the Hausdorff dimension of $X_{G}$ and a large class of similarly defined sets, putting it into the context of subshifts invariant under the semi-group of multiplicative integers [14, 15]. Here we adapt the techniques of [14, 15] to compute the full dimension spectrum $\operatorname{dim}_{H}\left(A_{\theta}\right)$.

Theorem 1. Let $A_{\theta}$ be given by (2). For $\theta \in(0,1)$ we have

$$
\operatorname{dim}_{H}\left(A_{\theta}\right)=f(\theta):=-\log _{2}(1-p)-\frac{\theta}{2} \log _{2}\left[\frac{(1-q)(1-p)}{q p}\right],
$$

where

$$
\begin{gathered}
p^{2} q=(1-p)^{3}, 0<p<1,0<q<1, \\
\theta=\frac{2 p(1-q)}{1+p+q} .
\end{gathered}
$$

We have $\operatorname{dim}_{H}\left(A_{0}\right)=\lim _{\theta \rightarrow 0} f(\theta)=-\log _{2}(1-p)$, with $p^{2}=(1-p)^{3}$, and $\operatorname{dim}_{H}\left(A_{1}\right)=$ $\lim _{\theta \rightarrow 1} f(\theta)=0$. 
The meaning of $p$ and $q$ will be explained in the next section. Of course, it is easy to eliminate $q$ from (3) and (5). For a given $\theta$, we get an algebraic equation of degree 4 for $p$. Solving the equation numerically yields the graph in Figure 1.

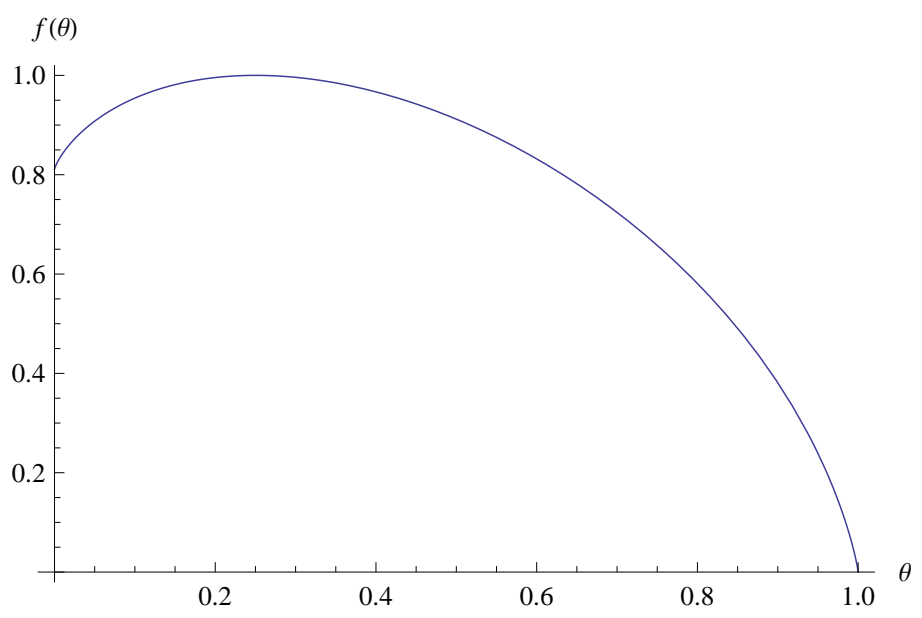

Figure 1. Dimension of $A_{\theta}$

\section{Remarks.}

1. As already mentioned, the formula for $\operatorname{dim}_{H}\left(A_{0}\right)$ easily follows from [14]. Note that in [14, 15] notation was slightly different, so that $p$ in those papers is $1-p$ here.

2. It is immediate that $A_{1}$ is contained in the set of $0-1$ sequences which have frequency of 1 's equal to 1 . Thus, $\operatorname{dim}_{H}\left(A_{1}\right)=0$ by (1), and we assume $\theta<1$ for the rest of the paper.

3. By the Strong Law of Large Numbers for weakly correlated random variables (see [18]), for a.e. sequence $\left(x_{k}\right)$ with respect to the Bernoulli $\left(\frac{1}{2}, \frac{1}{2}\right)^{\mathbb{N}}$ measure, $\lim _{n \rightarrow \infty} \frac{1}{n} \sum_{j=1}^{n} x_{j} x_{2 j}=1 / 4$. This agrees with our result: for $\theta=1 / 4$ we get $\operatorname{dim}_{H}\left(A_{\theta}\right)=1$ and $p=q=1 / 2$.

4. In [9] it is proved that $\operatorname{dim}_{H}\left(B_{\theta}\right)=1-\frac{1}{\ell}+\frac{1}{\ell} H\left(\frac{1+\theta}{2}\right)$ for $\theta \in[-1,1]$ and $\ell \geq 2$, where $B_{\theta}:=\left\{\left(x_{k}\right)_{1}^{\infty} \in\{-1,1\}^{\mathbb{N}}: \lim _{n \rightarrow \infty} \frac{1}{n} \sum_{k=1}^{n} x_{k} x_{2 k} \cdots x_{\ell k}=\theta\right\}$, using the techniques of Riesz products. It is further pointed out in [9] that the problem becomes drastically different if one takes the digits 0,1 (which reduces to $A_{\theta}$ for $\ell=2$ ) instead of $-1,1$.

5. Yu. Kifer [16] considered a slightly different question: he studied the Hausdorff dimension of sets defined by the frequencies of all $\ell$-tuples of digits $i_{1}, \ldots, i_{\ell}$ in positions $k, 2 k, \ldots, \ell k$. However, he was able to compute the dimensions only under the assumption that such frequencies are of the form $p_{i_{1}, \ldots, i_{\ell}}=p_{i_{1}} \cdots p_{i_{\ell}}$. 
6. As pointed out in [15], there are some parallels between the multiplicative shifts of finite type and self-affine carpets [3, 19]; we should also add here self-affine sponges [13. The present paper may similarly be compared to the work on multifractal self-affine carpets and sponges, see e.g. [17, 20, 1, 12]; however, we do not see any way to directly transfer the results.

\section{Preliminaries AND the SCheme of the Proof.}

The dimension of $A_{\theta}$ is computed with the help of the following lemma which goes back to Billingsley [5]. We write $[u]$ for the cylinder set of sequences starting with a finite word $u$ and $x_{1}^{n}:=x_{1} \ldots x_{n}$.

Lemma 2 (see Prop.4.9 in [8]). Let $E$ be a Borel set in $\Sigma_{2}$ and let $\nu$ be a finite Borel measure on $\Sigma_{2}$.

(i) If $\nu(E)>0$ and $\liminf _{n \rightarrow \infty} \frac{-\log _{2} \nu\left[x_{1}^{n}\right]}{n} \geq s$ for $\nu$-a.e. $x \in E$, then $\operatorname{dim}_{H}(E) \geq s$.

(ii) If $\liminf _{n \rightarrow \infty} \frac{-\log _{2} \nu\left[x_{1}^{n}\right]}{n} \leq s$ for all $x \in E$, then $\operatorname{dim}_{H}(E) \leq s$.

Following [14, 15], for a probability measure $\mu$ on $\Sigma_{2}$, we define another measure $\mathbb{P}_{\mu}$ on $\Sigma_{2}$ by

$$
\mathbb{P}_{\mu}[u]:=\prod_{i \leq n, i \text { odd }} \mu\left[\left.u\right|_{J(i)}\right], \text { where } J(i)=\left\{2^{r} i\right\}_{r=0}^{\infty}
$$

and $\left.u\right|_{J(i)}$ is the subsequence of $u$ (viewed as a finite sequence) along the geometric progression $J(i)$. The new measure $\mathbb{P}_{\mu}$ is invariant under the action of the multiplicative semigroup of odd positive numbers:

$$
\left(x_{k}\right)_{k=1}^{\infty} \mapsto\left(x_{i k}\right)_{k=1}^{\infty} \text { for odd } i
$$

We consider Markov measures $\mu_{\mathbf{p}, P}$ on $\Sigma_{2}$, with the initial probability distribution $\mathbf{p}=(1-p, p)$ (so that $p$ is the probability of initial 1$)$, and the stochastic transition matrix $P=\left(\begin{array}{cc}1-p & p \\ q & 1-q\end{array}\right)$. Note that our Markov measures are not stationary; instead, their initial distribution coincides with the first row of the transition matrix.

Next we indicate the scheme of the proof of Theorem 2 Recall that $\theta \in[0,1)$. In view of Lemma $2(\mathrm{i})$, the lower bound for $\operatorname{dim}_{H}\left(A_{\theta}\right)$ will be established once we prove the following.

Lemma 3. Fix $p \in(0,1), q \in[0,1)$, and let $\mathbb{P}_{\mu}$, with $\mu=\mu_{\mathbf{p}, P}$, be defined by (6).

(i) If $p, q$ satisfy (5), then $\mathbb{P}_{\mu}\left(\Sigma_{2} \backslash A_{\theta}\right)=0$. For $\theta=0$ we take $q=1$. 
(ii) For any $p, q$ we have

$$
\lim _{n \rightarrow \infty} \frac{-\log _{2} \mathbb{P}_{\mu}\left[x_{1}^{n}\right]}{n}=s(p, q):=\frac{(1+q) H(p)+p H(q)}{1+p+q} \text { for } \mathbb{P}_{\mu} \text {-a.e. } x .
$$

(iii) The maximum of $s(p, q)$, subject to (5), is achieved when $p^{2} q=(1-p)^{3}$, and it equals

$$
f(\theta)=-\log _{2}(1-p)-\frac{\theta}{2} \log _{2}\left[\frac{(1-q)(1-p)}{q p}\right]
$$

The upper bound in Theorem 1 will follow from Lemma 2(ii), once we prove the following

Lemma 4. Let $\mu=\mu_{\mathbf{p}, P}$ be the Markov measure with initial probability vector $\mathbf{p}=$ $(1-p, p)$ and transition matrix $P=\left(\begin{array}{cc}1-p & p \\ q & 1-q\end{array}\right)$, where $p^{2} q=(1-p)^{3}$ and $\quad$ 5) holds, and let $\mathbb{P}_{\mu}$ be the corresponding multiplicative invariant measure. Then

$$
\liminf _{n \rightarrow \infty} \frac{-\log _{2} \mathbb{P}_{\mu}\left[x_{1}^{n}\right]}{n} \leq f(\theta) \text { for all } x \in A_{\theta} .
$$

\section{Proofs}

The following elementary lemma will be useful. We provide the proof for completeness.

Lemma 5. Suppose that $\left\{z_{n}\right\}$ is a bounded real sequence and there exists $c>0$ such that

$$
\left|z_{n}-z_{n+m}\right| \leq \frac{c m}{n} \text { for all } m, n \in \mathbb{N} .
$$

If $z_{2^{k} n} \rightarrow \gamma$ as $k \rightarrow \infty$ for all $n \in \mathbb{N}$, then $z_{n} \rightarrow \gamma$.

Proof. For $\varepsilon>0$ let $i_{0} \in \mathbb{N}$ be such that $2^{-i_{0}}<\varepsilon$. By the assumption, we can find $k_{0} \in \mathbb{N}$ such that for all $\ell \leq 2^{i_{0}}$ and all $k \geq k_{0}-i_{0}$ we have $\left|z_{\ell \cdot 2^{k}}-\gamma\right|<\varepsilon$. For $n>2^{k_{0}+i_{0}}$, with $2^{k-1} \leq n<2^{k}$, let $\ell=\left\lceil n \cdot 2^{i_{0}-k}\right\rceil \leq 2^{i_{0}}$. Then $0 \leq \ell \cdot 2^{k-i_{0}}-n<2^{k-i_{0}}$, hence

$$
\left|z_{n}-\gamma\right| \leq\left|z_{n}-z_{\ell \cdot 2^{k-i_{0}}}\right|+\left|z_{\ell \cdot 2^{k-i_{0}}}-\gamma\right| \leq \frac{c 2^{k-i_{0}}}{n}+\varepsilon<\varepsilon(1+2 c),
$$

completing the proof.

For positive integers $m<n, i, j \in\{0,1\}$, and $x \in \Sigma_{2}$ let

$$
\begin{gathered}
N_{i}\left(x_{m}^{n}\right)=\#\left\{k \in[m, n] \cap \mathbb{N}: x_{k}=i\right\}, \\
N_{i j}\left(x_{m}^{n}\right)=\#\left\{k \in[m, n] \cap \mathbb{N}: x_{k}=i, x_{2 k}=j\right\} .
\end{gathered}
$$


Further, for $x \in \Sigma_{2}$ and even $n$ denote

$$
\alpha_{n}(x)=\frac{N_{1}\left(x_{n / 2+1}^{n}\right)}{n / 2} .
$$

Observe that $\alpha_{n}(x) \in[0,1]$, and it is easy to see that

$$
\left|\alpha_{n}-\alpha_{n+m}\right| \leq \frac{2 m}{n} \text { for } n, m \text { even. }
$$

Lemma 6. Let $\mu$ be a Markov measure on $\Sigma_{2}$, with initial probability (row) vector $\mathbf{p}=$ $(1-p, p)$ and transition matrix $P=\left(\begin{array}{cc}1-p & p \\ q & 1-q\end{array}\right)$. Let $\mathbb{P}_{\mu}$ be the measure on $\Sigma_{2}$ defined by (6). Then for $\mathbb{P}_{\mu}$-a.e. $x \in \Sigma_{2}$ we have

$$
\lim _{n \rightarrow \infty} \alpha_{n}(x)=\frac{2 p}{1+p+q}=: \xi
$$

where $\alpha_{n}(x)$ is defined in (10).

Proof. We assume $n$ to be even. Consider $n \alpha_{2 n}(x)$, the number of 1's in $x_{i}$ 's for $i \in$ $(n, 2 n]$. There are $n / 2$ odd numbers $i$ in $(n, 2 n]$. By the definition of the measure $\mathbb{P}_{\mu}$, the corresponding $x_{i}$ are chosen to be 1 independently with probability $p$. Thus, by the Law of Large Numbers, the number of 1's among them is $n p / 2+o(n)$. Among the even numbers $2 i$ in $(n / 2, n]$, there is $n \alpha_{n}(x) / 2$ numbers for which $x_{i}=1$, and there will be approximately $n \alpha_{n}(x)(1-q) / 2$ 1's there, again using the Law of Large Numbers, since $1-q$ is the probability of the transition $1 \rightarrow 1$ according to the Markov measure $\mu$. Similarly, there is $n\left(1-\alpha_{n}(x)\right) / 2$ numbers $2 i \in(n, 2 n]$ for which $x_{i}=0$, and approximately $n\left(1-\alpha_{n}(x)\right) p / 2$ 's among those. Hence we have

$$
\begin{aligned}
\alpha_{2 n}(x) & =\frac{p}{2}+\frac{\alpha_{n}(x)(1-q)}{2}+\frac{\alpha_{n}(x)\left(1-\alpha_{n}(x)\right) p}{2}+\varepsilon_{n}(x) \\
& =p+\frac{\alpha_{n}(x)(1-q-p)}{2}+\varepsilon_{n}(x),
\end{aligned}
$$

where $\varepsilon_{n}(x) \rightarrow 0$ for $\mathbb{P}_{\mu}$-a.e. $x$. Since $|(1-q-p) / 2|<1$, it follows that $\alpha_{2^{k} n}(x) \rightarrow \xi$ for $\mathbb{P}_{\mu}$-a.e. $x$, where $\xi=p+\xi(1-q-p) / 2$, i.e. $\xi=\frac{2 p}{1+p+q}$. Then for $\mathbb{P}_{\mu}$-a.e. $x$ we have $\alpha_{2^{k} n}(x) \rightarrow \xi$ for all $n \in \mathbb{N}$. It remains to recall (11) and apply Lemma 5 .

Proof of Lemma 3(i). We claim that

$$
\lim _{n \rightarrow \infty} \frac{N_{11}\left(x_{1}^{n}\right)}{n}=\xi(1-q)=\frac{2 p(1-q)}{1+p+q}=\theta
$$

for a $\mathbb{P}_{\mu}$-typical point $x \in \Sigma_{2}$. Indeed, the initial part of the sequence does not impact the limit, and for $n$ big enough we see 1's with frequency $\xi$ and thus 11's (in positions 
$i, 2 i)$ with frequency $\xi(1-q)$, by the definition of $\mathbb{P}_{\mu}$. This means that $\mathbb{P}_{\mu}$-a.e. $x$ is in $A_{\theta}$, as desired.

Fix an even integer $n$. Denote

$$
N_{1, \text { odd }}=N_{1, \text { odd }}\left(x_{1}^{n}\right):=\#\left\{k \leq n: k \text { odd }, x_{k}=1\right\} .
$$

By the definition of $\mu=\mu_{\mathbf{p}, P}$ and $\mathbb{P}_{\mu}$ we have, for any $x$ and even $n$ :

$$
\mathbb{P}_{\mu}\left[x_{1}^{n}\right]=p^{N_{1, \text { odd }}}(1-p)^{n / 2-N_{1, \text { odd }}}(1-p)^{N_{00}} p^{N_{01}} q^{N_{10}}(1-q)^{N_{11}}
$$

where $N_{i j}=N_{i j}\left(x_{1}^{n / 2}\right)$.

For the rest of the proof we write log for $\log _{2}$ to simplify notation.

Proof of Lemma 3(ii). For $\mathbb{P}_{\mu}$-a.e. $x \in \Sigma_{2}$, we see 1's with frequency $p$ in odd places, and similarly to the proof of Lemma 3(i), the frequency of 00's, 01's, 10's, and 11's is $(1-\xi)(1-p),(1-\xi) p, \xi q$, and $\xi(1-q)$ respectively. Therefore, for $\mathbb{P}_{\mu}$-a.e. $x \in \Sigma_{2}$ the formula 12 yields

$$
\begin{aligned}
\lim _{n \rightarrow \infty} \frac{-\log \mathbb{P}_{\mu}\left[x_{1}^{n}\right]}{n}= & -(1 / 2)[(2-\xi) p \log p+(2-\xi)(1-p) \log (1-p) \\
& +\xi q \log q+\xi(1-q) \log (1-q)] \\
= & \frac{(1+q) H(p)+p H(q)}{1+p+q},
\end{aligned}
$$

as desired.

In view of Lemma 3(i),(ii) and Lemma 2(i), we have that

$$
\operatorname{dim}_{H}\left(A_{\theta}\right) \geq s(p, q)=\frac{(1+q) H(p)+p H(q)}{1+p+q}, \text { where } \theta=\frac{2 p(1-q)}{1+p+q} .
$$

Thus, we should find the constrained maximum of $s(p, q)$ on $[0,1]^{2}$. This is a straightforward exercise, but we include it for the record and in order to explain where the formula (4) comes from. It is actually not needed for the proof of the main result, since we could just produce the answer for the optimization problem and refer to Lemma 4. We also include the verification of (7).

Proof of Lemma 3(iii). We use the method of Lagrange multipliers. Differentiating $s(p, q)$ yields

$$
\begin{aligned}
(1+p+q)^{2} \frac{\partial s(p, q)}{\partial p} & =(1+q)\left[(1+p+q) \log \left(\frac{1-p}{p}\right)-H(p)+H(q)\right] \\
& =(1+q)[(2+q) \log (1-p)-(1+q) \log p+H(q)],
\end{aligned}
$$




$$
\begin{aligned}
(1+p+q)^{2} \frac{\partial s(p, q)}{\partial q} & =p\left[(1+p+q) \log \left(\frac{1-q}{q}\right)+H(p)-H(q)\right] \\
& =p[(2+p) \log (1-q)-(1+p) \log q+H(p)] .
\end{aligned}
$$

Differentiating the constraint $g(p, q)=\theta(1+p+q)-2 p(1-q)=0$ yields

$$
\nabla g(p, q)=(\theta-2(1-q), \theta+2 p)=\left(\frac{-2(1-q)(1+q)}{1+p+q}, \frac{2 p(2+p)}{1+p+q}\right) .
$$

At the point of constrained maximum we have $\nabla s(p, q)=\lambda \nabla g(p, q)$, which reduces to

$$
\begin{aligned}
& (2+p)[(2+q) \log (1-p)-(1+q) \log p+H(q)] \\
= & -(1-q)[(2+p) \log (1-q)-(1+p) \log q+H(p)] .
\end{aligned}
$$

The latter becomes, after collecting the terms:

$$
3(1+p+q) \log (1-p)=2(1+p+q) \log p+(1+p+q) \log q
$$

so $p^{2} q=(1-p)^{3}$, as claimed.

It remains to verify the formula (7). We have

$$
\begin{aligned}
f(\theta) & =-\log (1-p)-\frac{\theta}{2} \log \left[\frac{(1-q)(1-p)}{q p}\right] \\
& =-\log (1-p)-\frac{p(1-q)}{1+p+q} \log \left[\frac{(1-q)(1-p)}{q p}\right] .
\end{aligned}
$$

Comparing the latter with

$$
s(p, q)=\frac{\left(p \log \frac{1-p}{p}-\log (1-p)\right)(1+q)+p\left(q \log \frac{1-q}{q}-\log (1-q)\right)}{1+p+q}
$$

results in $(1+p+q)(f(\theta)-s(p, q))=-p\left[2 \log \frac{1-p}{p}+\log \frac{1-q}{q}+\log (1-p)-\log (1-q)\right]=0$, whenever $p^{2} q=(1-p)^{3}$, as desired.

Proof of Lemma 4. Fix any $x$ and an even $n \in \mathbb{N}$. We continue to use the notation $N_{i j}=N_{i j}\left(x_{1}^{n / 2}\right)$. Since $N_{00}+N_{01}+N_{10}+N_{11}=n / 2$, we have from 12 :

$$
\begin{aligned}
\mathbb{P}_{\mu}\left[x_{1}^{n}\right] & =p^{N_{1, \text { odd }}+N_{01}}(1-p)^{n-N_{1, \text { odd }}-N_{01}-N_{10}-N_{11}} q^{N_{10}}(1-q)^{N_{11}} \\
& =(1-p)^{n}\left(\frac{p}{1-p}\right)^{N_{1, \text { odd }}+N_{01}}\left(\frac{q}{1-q}\right)^{N_{10}}\left(\frac{1-q}{1-p}\right)^{N_{10}+N_{11}}
\end{aligned}
$$

Observe that

$$
N_{10}+N_{11}=N_{1}\left(x_{1}^{n / 2}\right) \quad \text { and } \quad N_{1, \text { odd }}=N_{1}\left(x_{1}^{n}\right)-N_{01}-N_{11}
$$


The equation $p^{2} q=(1-p)^{3}$ can be rewritten as $\frac{1-q}{1-p}=\frac{1-q}{q}\left(\frac{1-p}{p}\right)^{2}$. Combining this with the last several equalities yields

$$
\begin{aligned}
\mathbb{P}_{\mu}\left[x_{1}^{n}\right] & =(1-p)^{n}\left(\frac{p}{1-p}\right)^{N_{1}\left(x_{1}^{n}\right)-N_{11}-2 N_{1}\left(x_{1}^{n / 2}\right)}\left(\frac{1-q}{q}\right)^{N_{11}} \\
& =(1-p)^{n}\left(\frac{(1-q)(1-p)}{q p}\right)^{N_{11}}\left(\frac{p}{1-p}\right)^{N_{1}\left(x_{1}^{n}\right)-2 N_{1}\left(x_{1}^{n / 2}\right)} .
\end{aligned}
$$

Thus,

$$
\begin{aligned}
\frac{-\log \mathbb{P}_{\mu}\left[x_{1}^{n}\right]}{n}= & -\log (1-p)-\frac{N_{11}\left(x_{1}^{n / 2}\right)}{n} \log \left(\frac{(1-q)(1-p)}{q p}\right) \\
& +\left(\frac{N_{1}\left(x_{1}^{n}\right)}{n}-\frac{N_{1}\left(x_{1}^{n / 2}\right)}{n / 2}\right) \log \left(\frac{p}{1-p}\right) \\
= & f(\theta)+\left(\theta-\frac{N_{11}\left(x_{1}^{n / 2}\right)}{n}\right) \log \left(\frac{(1-q)(1-p)}{q p}\right) \\
& +\left(\frac{N_{1}\left(x_{1}^{n}\right)}{n}-\frac{N_{1}\left(x_{1}^{n / 2}\right)}{n / 2}\right) \log \left(\frac{p}{1-p}\right),
\end{aligned}
$$

where $f(\theta)$ is from (7). Observe that $\lim _{n \rightarrow \infty} \frac{N_{11}\left(x_{1}^{n / 2}\right)}{n}=\theta / 2$ for all $x \in A_{\theta}$. Now replace $n$ by $2^{\ell}$ for $\ell=1, \ldots, L$, and take the average over $\ell$. The expression in the last line of (14) "telescopes," so we obtain

$$
\begin{aligned}
\frac{1}{L} \sum_{\ell=1}^{L}\left(\frac{-\log \mathbb{P}_{\mu}\left[x_{1}^{2^{\ell}}\right]}{2^{\ell}}-f(\theta)\right) & =\frac{1}{L} \sum_{\ell=1}^{L}\left(\frac{\theta}{2}-\frac{N_{11}\left(x_{1}^{2^{\ell}}\right)}{2^{\ell}}\right) \log \left(\frac{(1-q)(1-p)}{q p}\right) \\
& +\frac{1}{L} \log \left(\frac{p}{1-p}\right)\left(\frac{N_{1}\left(x_{1}^{2^{L}}\right)}{2^{L}}-\frac{N_{1}\left(x_{1}^{2}\right)}{2}\right)
\end{aligned}
$$

which tends to zero, as $L \rightarrow \infty$. It follows that

$$
\liminf _{\ell \rightarrow \infty} \frac{-\log \mathbb{P}_{\mu}\left[x_{1}^{2^{\ell}}\right]}{2^{\ell}} \leq f(\theta) \text { for all } x \in A_{\theta},
$$

and the proof of $(8)$ is complete.

\section{Concluding Remarks}

1. It is not hard to verify that, under the conditions $\sqrt{4}$ and 5 we have $\frac{(1-q)(1-p)}{q p}<1$ if and only if $\theta<1 / 4$. Therefore, by the argument in the last section, it immediately follows that

$$
\operatorname{dim}_{H}\left(A_{\theta}^{+}\right)=f(\theta) \text { for } \theta \in(0,1 / 4), \quad \operatorname{dim}_{H}\left(A_{\theta}^{-}\right)=f(\theta) \text { for } \theta \in(1 / 4,1),
$$


where

$$
\begin{aligned}
& A_{\theta}^{+}:=\left\{\left(x_{k}\right)_{1}^{\infty} \in \Sigma_{2}: \limsup _{n \rightarrow \infty} \frac{1}{n} \sum_{k=1}^{n} x_{k} x_{2 k} \leq \theta\right\}, \\
& A_{\theta}^{-}:=\left\{\left(x_{k}\right)_{1}^{\infty} \in \Sigma_{2}: \liminf _{n \rightarrow \infty} \frac{1}{n} \sum_{k=1}^{n} x_{k} x_{2 k} \geq \theta\right\} .
\end{aligned}
$$

2. We extended the result of Theorem 1 to the case of arbitrary functions $f_{1}, f_{2}$ on the shift $\Sigma_{2}$ depending on the first digit $x_{1}$. The method is the same, but the calculations are more involved, so we only state the result.

Theorem 7. For $\beta, \gamma \in \mathbb{R}$, let

$$
A_{\theta}(\beta, \gamma):=\left\{\left(x_{k}\right)_{1}^{\infty} \in \Sigma_{2}: \lim _{n \rightarrow \infty} \frac{1}{n} \sum_{k=1}^{n}\left(x_{k}+\beta\right)\left(x_{2 k}+\gamma\right)=\theta\right\}
$$

We have

$$
\operatorname{dim}_{H}\left(A_{\theta}(\beta, \gamma)\right)=-\frac{1}{2} \log _{2}\left[p_{0}(1-p)\right]-\frac{\theta}{2} \log _{2}\left[\frac{(1-q)(1-p)}{q p}\right]
$$

where

$$
\frac{1-p}{1-q}=\left(\frac{q}{1-q}\right)^{1+2 \beta+\gamma}\left(\frac{p}{1-p}\right)^{2+2 \beta+\gamma}, \quad \frac{1-p_{0}}{p_{0}}=\left(\frac{q}{1-q}\right)^{\beta}\left(\frac{p}{1-p}\right)^{1+\beta},
$$

and

$$
\theta=\beta \gamma+\frac{(1+\beta+\gamma-q)\left(1+p-p_{0}\right)+\beta\left(p_{0}(p+q)-q\right)}{1+p+q} .
$$

The appropriate measure is $\mathbb{P}_{\mu}$, with $\mu$ Markov, having the initial distribution $\left(p_{0}, 1-p_{0}\right)$ and the transition matrix $\left(\begin{array}{cc}1-p & p \\ q & 1-q\end{array}\right)$.

3. After this work was essentially completed, we were informed that A.-H. Fan, J. Schmeling, and M. Wu have computed the dimension of $A_{\theta}$ (in a different, but equivalent form) and other sets of this type, independently, but also building on [15.

Acknowledgment. We are grateful to the referee for helpful comments and suggestions.

\section{REFERENCES}

[1] J. Barral and M. Mensi, Multifractal analysis of Birkhoff averages on 'self-affine' symbolic spaces, Nonlinearity, 21(2008), no. 10, 2409-2425.

[2] L. Barreira, Dimension and recurrence in hyperbolic dynamics. Progress in Mathematics, 272, Birkhäuser Verlag, Basel, (2008).

[3] T. Bedford, Crinkly curves, Markov partitions and box dimension in self-similar sets, Ph.D. Thesis, University of Warwick, (1984). 
[4] A. S. Besicovitch, On the sum of digits of real numbers represented in the dyadic system, Math. Annalen, 110(1934), 321-330.

[5] P. Billingsley, Ergodic theory and information, Wiley, New York, (1965).

[6] J. Bourgain, Double recurrence and almost sure convergence, J. Reine Angew. Math., 404(1990), 140-161.

[7] H. G. Eggleston, The fractional dimension of a set defined by decimal properties, Quart. J. Math. 20(1949), 31-36.

[8] K. Falconer, Fractal geometry. Mathematical foundations and applications, John Wiley \& Sons, Chichester, (1990).

[9] A. Fan, L. Liao, J. Ma, Level sets of multiple ergodic averages, Preprint arXiv:1105.3032, to appear in Monatsh. Math.

[10] H. Furstenberg, Ergodic behavior of diagonal measures and a theorem of Szemerédi on arithmetic progressions, J. d'Analyse Math. 31(1977), 204-256.

[11] B. Host and B. Kra, Nonconventional ergodic averages and nilmanifolds, Ann. Math. 161(2005), 397-488.

[12] T. Jordan and M. Rams, Multifractal analysis for Bedford-McMullen carpets, Math. Proc. Camb. Phil. Soc. 150(2011), 147-156.

[13] R. Kenyon and Y. Peres, Measures of full dimension on affine-invariant sets, Ergodic Theory and Dynamical Systems, 16(1996), 307-323.

[14] R. Kenyon, Y. Peres, and B. Solomyak, Hausdorff dimension of the multiplicative golden mean shift, C. R. Math. Acad. Sci. Paris 349(2011), 625-628.

[15] R. Kenyon, Y. Peres, and B. Solomyak, Hausdorff dimension for fractals invariant under the multiplicative integers, Preprint arXiv 1102.5136, to appear in Ergodic Theory and Dynamical Systems.

[16] Yu. Kifer, A nonconventional strong law of large numbers and fractal dimensions of some multiple recurrence sets, Preprint arXiv:1012.2799

[17] J. King, The singularity spectrum for general Sierpiński carpets, Adv. Math. 116(1995), 1-8.

[18] R. Lyons, Strong laws of large numbers for weakly correlated random variables, Michigan Math. J. 35(1988), 353-359.

[19] C. McMullen, The Hausdorff dimension of general Sierpinski carpets, Nagoya Math. J. 96(1984), 1-9.

[20] L. Olsen, Self-affine multifractal Sierpinski sponges in $\mathbb{R}^{d}$, Pacific J. Math. 183(1998), no. 1, $143-199$.

Yuval Peres, One Microsoft Way, Redmond, WA 98052, USA

E-mail address: peres@icrosoft.com

Boris Solomyak, Box 354350, Department of Mathematics, University of Washington, SEATTLE WA 98195, USA

E-mail address: solomyak@math.washington.edu 